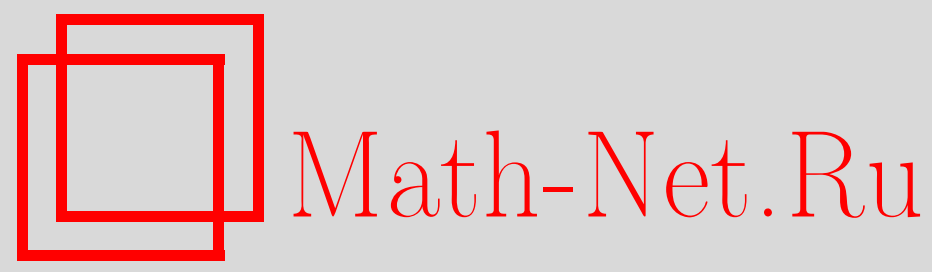

А. М. Зубков, А. А. Серов, Оценка числа булевых функций, имеющих аффинные приближения заданной точности, Дискрет. матем., 2010, том 22, выпуск 4, 3-19

DOI: https://doi.org/10.4213/dm1115

Использование Общероссийского математического портала Math-Net.Ru подразумевает, что вы прочитали и согласны с пользовательским соглашением http://www . mathnet.ru/rus/agreement

Параметры загрузки:

IP: 54.198 .64 .247

26 апреля 2023 г., 16:04:32 


\title{
Оценка числа булевых функций, имеющих аффинные приближения заданной точности
}

\author{
() 2010 г. А. М. Зубков, А. А. Серов
}

\begin{abstract}
Получены двусторонние оценки и асимптотические формулы для числа булевых функций от $n$ переменных, которые с заданной точностью аппроксимируются аффинными или линейными булевыми функциями.

Работа выполнена при поддержке Российского фонда фундаментальных исследований, проект 08-01-00078.
\end{abstract}

\section{1. Введение}

Пусть $\mathbf{F}_{2}-$ поле из двух элементов. Для произвольного натурального числа $n$ будем обозначать через $V_{n}=\mathbf{F}_{2}^{n}$ пространство $n$-мерных векторов с компонентами из $\mathbf{F}_{2}$. Между множеством $\mathbf{F}_{2}^{V_{n}}=\left\{f: V_{n} \rightarrow \mathbf{F}_{2}\right\}$ всех булевых функций от $n$ переменных и пространством $V_{2^{n}}$ можно установить взаимно однозначное соответствие, отождествляя функцию $f \in \mathbf{F}_{2}^{V_{n}}$ с вектором $\left\{f(x) \mid x \in V_{n}\right\}$.

Расстояние Хемминга $\rho(f, g)$ между булевыми функциями $\left\{f, g \in \mathbf{F}_{2}^{V_{n}}\right\}$ определяется как число значений переменной $x \in V_{n}$, при которых $f(x) \neq g(x)$. Для произвольного множества булевых функций $A \subset \mathbf{F}_{2}^{V_{n}}$ и функции $f \in \mathbf{F}_{2}^{V_{n}}$ обозначим через

$$
\rho(f, A)=\min _{g \in A} \rho(f, g)
$$

расстояние Хэмминга от $f$ до ближайшей к ней функции из множества $A$.

В множестве $\mathbf{F}_{2}^{V_{n}}$ всех булевых функций естественно выделяется класс аффинных функций

$$
\mathbf{A}_{n}=\left\{f \in \mathbf{F}_{2}^{V_{n}}: f\left(x_{1}, \ldots, x_{n}\right)=a_{0} \oplus a_{1} x_{1} \oplus \ldots \oplus a_{n} x_{n}, a_{0}, \ldots, a_{n} \in \mathbf{F}_{2}\right\},
$$

где $\oplus-$ сложение в $\mathbf{F}_{2}$. Множество $\mathbf{A}_{n}$ состоит из $\left|\mathbf{A}_{n}\right|=2^{n+1}$ элементов. Занумеруем их следующим образом:

$$
l_{j}(x)=a_{0} \oplus a_{1} x_{1} \oplus \ldots \oplus a_{n} x_{n}, \quad \text { если } \quad j=\sum_{k=0}^{n} a_{k} 2^{k} .
$$

При такой нумерации $l_{0}(x) \equiv 0, l_{1}(x) \equiv 1, l_{2^{k}}(x)=x_{k}, k=1,2, \ldots, n$, и $l_{2 j}(x) \oplus l_{2 j+1}(x)=1, x \in V_{n}, j=0, \ldots, 2^{n}-1$, поэтому

$$
\rho\left(l_{i}, l_{j}\right)=2^{n-1}, \quad \text { если } i \neq j,\{i, j\} \notin \bigcup_{k=0}^{2^{n}-1}\{2 k, 2 k+1\} .
$$


Пусть

$$
\mathbf{F}_{2}^{V_{n}}(r)=\left\{f \in \mathbf{F}_{2}^{V_{n}}: \rho\left(f, \mathbf{A}_{n}\right) \leqslant r\right\}
$$

- множество всех булевых функций от $n$ переменных, расстояние от которых до множества $\mathbf{A}_{n}$ аффинных функций меньше или равно $r<2^{n-1}$. Известно, что

$$
\mathbf{F}_{2}^{V_{n}}(r)=\mathbf{F}_{2}^{V_{n}}, \quad r \geqslant 2^{n-1}-2^{n / 2-1} .
$$

При четном $n$ функции, расстояние от которых до множества аффинных функций равно $2^{n-1}-2^{n / 2-1}$, называются бент-функциями или максимально-нелинейными функциями. Они, в частности, представляют интерес для построения криптографических алгоритмов, стойких по отношению к методам, основанным на аппроксимации булевых функций линейными (см., например, [1]). Однако даже асимптотика числа бент-функций до сих пор неизвестна.

Бент-функции образуют экстремальный класс булевых функций, наиболее удаленных от аффинных функций. Представляет интерес исследование других классов булевых функций, например, классов функций, расстояния от которых до множества аффинных функций не превосходят заданной величины.

Предельные теоремы для распределения расстояния от случайной булевой функции до множества линейных булевых функций доказаны в [2], а в [4] доказаны предельные теоремы для распределения расстояния от случайной булевой функции до множества квадратичных булевых форм со свободным членом. В частности, в [2] показано, что если $\mathbf{F}_{2}^{V_{n}, 0}(r)$ - множество всех булевых функций, расстояние Хемминга от которых до множества линейных функций (аффинных с нулевым свободным членом $a_{0}$ ) не превосходит $r$, то при $n \rightarrow \infty$

$$
\sup _{0 \leqslant r<2^{n-1}-2^{n / 2-1}}\left|2^{-2^{n}}\right| \mathbf{F}_{2}^{V_{n}, 0}(r)\left|-\left(1-e^{-e^{-x(n, r)}}\right)\right| \rightarrow 0
$$

где $x(n, r)$ определяется равенством

$$
r=2^{n-1}-\sqrt{2^{n-1}\left(n \ln 2-\frac{1}{2} \ln (4 \pi n \ln 2)+x(n, r)\right)} .
$$

Это означает, что для основной массы булевых функций от $n$ переменных расстояние до множества линейных функций при $n \rightarrow \infty$ имеет вид

$$
2^{n-1}-\sqrt{2^{n-1}\left(n \ln 2-\frac{1}{2} \ln n\right)}+O\left(\sqrt{2^{n} / n}\right) .
$$

В настоящей работе получены точные формулы и верхние и нижние оценки для чисел элементов множеств $\mathbf{F}_{2}^{V_{n}}(r)$ и $\mathbf{F}_{2}^{V_{n}, 0}(r)$. Один из основных результатов (объединение части формулировок теорем 2, 3, 5 и предложений 1,2) можно сформулировать следующим образом.

Теорема 1. Если $n \geqslant 2$, то

$$
\begin{aligned}
& (1-Q(n, r)) 2^{n+1} \sum_{m=0}^{r}\left(\begin{array}{l}
2^{n} \\
m
\end{array}\right) \leqslant\left|\mathbf{F}_{2}^{V_{n}}(r)\right| \leqslant 2^{n+1} \sum_{m=0}^{r}\left(\begin{array}{l}
2^{n} \\
m
\end{array}\right), \\
& \left(1-\frac{1}{2} Q(n, r)\right) 2^{n} \sum_{m=0}^{r}\left(\begin{array}{l}
2^{n} \\
m
\end{array}\right) \leqslant\left|\mathbf{F}_{2}^{V_{n}, 0}(r)\right| \leqslant 2^{n} \sum_{m=0}^{r}\left(\begin{array}{l}
2^{n} \\
m
\end{array}\right),
\end{aligned}
$$


где $Q(n, r)=0$ при $0 \leqslant r<2^{n-2}$,

$$
Q(n, r)<\frac{1}{15} 2^{-\left(c_{r}^{2}-3 / 2\right) n} \exp \left\{\frac{2\left(c_{r}^{2} n\right)^{3 / 2}}{2^{n / 2}}\right\}
$$

nри $n \geqslant 8, r=2^{n-1}-c_{r} \sqrt{2^{n-1} n \ln 2} \geqslant 0, c_{r}>1$.

Отношения верхних и нижних оценок в теореме 1 стремятся к 1 , если $n \rightarrow \infty$ и $r<2^{n-1}-c \sqrt{2^{n-1} n \ln 2}, c>\sqrt{3 / 2}=1,2247 \ldots$

Для сумм $\sum_{m=0}^{r}\left(\begin{array}{c}2^{n} \\ m\end{array}\right)$ при $r<2^{n-1}$ в Разделе 2 получены явные верхние и нижние

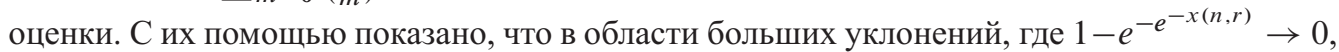
относительная погрешность приближения величины $2^{-2^{n}}\left|\mathbf{F}_{2}^{V_{n}, 0}(r)\right|$ величиной $1-e^{-e^{-x(n, r)}}$ быстро растет с уменьшением $r$.

Следствие 1. Если $\varepsilon>0 u n, r \rightarrow \infty$ так, что выполняется условие

$$
r<2^{n-1}-(1+\varepsilon) \sqrt{2^{n-1} n \ln 2},
$$

то при достаточно больших $n$

$$
\frac{\left|\mathbf{F}_{2}^{V_{n}, 0}(r)\right|}{2^{2^{n}}\left(1-e^{-e^{-x(n, r)}}\right)}<1,25 \frac{\exp \left\{2\left(1-r / 2^{n-1}\right)\right\}}{2^{n / 2}\left(1-r / 2^{n-1}\right)} \sqrt{n} .
$$

\section{2. Неравенства включения-исключения}

Считая равными нулю суммы, в которых верхний предел суммирования меньше нижнего, введем обозначения

$$
\begin{aligned}
N_{1}(n, r)= & \sum_{m=0}^{r}\left(\begin{array}{c}
2^{n} \\
m
\end{array}\right), \\
N_{2}(n, r)= & \sum_{m_{0}=0}^{r-2^{n-2}}\left(\begin{array}{c}
2^{n-1} \\
m_{0}
\end{array}\right) \sum_{m_{1}=2^{n-1}-\left(r-m_{0}\right)}^{r-m_{0}}\left(\begin{array}{c}
2^{n-1} \\
m_{1}
\end{array}\right), \\
S(a, b)= & \sum_{\substack{g, h \geqslant 0: \\
g+h \leqslant a, g-h \mid \leqslant b}}\left(\begin{array}{c}
2^{n-2} \\
g
\end{array}\right)\left(\begin{array}{c}
2^{n-2} \\
h
\end{array}\right), \\
N_{3}(n, r)= & \sum_{v=0}^{r-2^{n-2}} \sum_{u=2^{n-1}-r+2 v}^{r}\left(\begin{array}{c}
2^{n-2} \\
v
\end{array}\right)\left(\begin{array}{c}
2^{n-2} \\
u-v
\end{array}\right) S\left(r-u, r+u-2 v-2^{n-1}\right) .
\end{aligned}
$$

Теорема 2. (a) Если $0 \leqslant r<2^{n-1}-2^{n / 2-1}$, по

$$
\begin{aligned}
\left|\mathbf{F}_{2}^{V_{n}}(r)\right| & \leqslant 2^{n+1} N_{1}(n, r), \\
2^{n+1} N_{1}(n, r)-4\left(\begin{array}{c}
2^{n} \\
2
\end{array}\right) N_{2}(n, r) & \leqslant\left|\mathbf{F}_{2}^{V_{n}}(r)\right| \\
& \leqslant 2^{n+1} N_{1}(n, r)-4\left(\begin{array}{c}
2^{n} \\
2
\end{array}\right) N_{2}(n, r)+8\left(\begin{array}{c}
2^{n} \\
3
\end{array}\right) N_{3}(n, r) .
\end{aligned}
$$


(б) Если $0 \leqslant r<2^{n-2}$, то

$$
\left|\mathbf{F}_{2}^{V_{n}}(r)\right|=2^{n+1} N_{1}(n, r) .
$$

(в) Если $2^{n-2} \leqslant r<2^{n-2}+2^{n-4}$, то $\left|\mathbf{F}_{2}^{V_{n}}(r)\right|$ равно правой части последнего неравенства в (3).

Теорема 3. (a) Если $0 \leqslant r<2^{n-1}-2^{n / 2-1}$, по

$$
\begin{aligned}
\left|\mathbf{F}_{2}^{V_{n}, 0}(r)\right| & \leqslant 2^{n} N_{1}(n, r), \\
2^{n} N_{1}(n, r)-\left(\begin{array}{c}
2^{n} \\
2
\end{array}\right) N_{2}(n, r) & \leqslant\left|\mathbf{F}_{2}^{V_{n}, 0}(r)\right| \\
& \leqslant 2^{n} N_{1}(n, r)-\left(\begin{array}{c}
2^{n} \\
2
\end{array}\right) N_{2}(n, r)+\left(\begin{array}{c}
2^{n} \\
3
\end{array}\right) N_{3}(n, r) .
\end{aligned}
$$

(б) Если $0 \leqslant r<2^{n-2}$, то

$$
\left|\mathbf{F}_{2}^{V_{n}, 0}(r)\right|=2^{n} N_{1}(n, r)
$$

(в) Если $2^{n-2} \leqslant r<2^{n-2}+2^{n-4}$, то $\left|\mathbf{F}_{2}^{V_{n}, 0}(r)\right|$ равно правой части последнего неравенства в (4).

Доказательство теоремь 2. Так как

$$
\mathbf{F}_{2}^{V_{n}}(r)=\bigcup_{j=0}^{2^{n+1}-1}\left\{f \in \mathbf{F}_{2}^{V_{n}}: \rho\left(f, l_{j}\right) \leqslant r\right\}
$$

то согласно формуле включения-исключения [3]

$$
\left|\mathbf{F}_{2}^{V_{n}}(r)\right|=\sum_{k=1}^{2^{n+1}}(-1)^{k-1} \sum_{0 \leqslant i_{1}<\ldots<i_{k}<2^{n+1}}\left|\left\{f \in \mathbf{F}_{2}^{V_{n}}: \max _{1 \leqslant j \leqslant k} \rho\left(f, l_{i_{j}}\right) \leqslant r\right\}\right|,
$$

а значения неполных сумм по $k$ от 1 до $m=1,2, \ldots, 2^{n+1}$ дают верхние (при нечетных $m$ ) и нижние (при четных $m$ ) оценки для $\left|\mathbf{F}_{2}^{V_{n}}(r)\right|$. Поэтому для доказательства теоремы достаточно получить формулы для чисел и значений слагаемых в правой части (5), соответствующих $k=1,2,3$, и показать, что при $r<2^{n-2}+2^{n-4}$ не существует булевых функций $f$, находящихся в $r$-окрестности четырех (а, значит, и любого числа $k>4)$ разных аффинных функций.

При $k=1$ слагаемые в (5) имеют вид

$$
\left|\left\{f \in \mathbf{F}_{2}^{V_{n}}: \rho\left(f, l_{j}\right) \leqslant r\right\}\right|=\sum_{m=0}^{r}\left(\begin{array}{l}
2^{n} \\
m
\end{array}\right)=N_{1}(n, r) .
$$

Число таких слагаемых в (5) равно $2^{n+1}$. В силу (1) и неравенства треугольника, множества $\left\{f \in \mathbf{F}_{2}^{V_{n}}: \rho\left(f, l_{j}\right) \leqslant r\right\}, 0 \leqslant j<2^{n+1}$, при $r<2^{n-2}$ не пересекаются. Поэтому при $r<2^{n-2}$ все слагаемые в (5) с $k>1$ равны 0 и

$$
\left|\mathbf{F}_{2}^{V_{n}}(r)\right|=\sum_{j=0}^{2^{n+1}-1}\left|\left\{f \in \mathbf{F}_{2}^{V_{n}}: \rho\left(f, l_{j}\right) \leqslant r\right\}\right|=2^{n+1} \sum_{m=0}^{r}\left(\begin{array}{l}
2^{n} \\
m
\end{array}\right)=2^{n+1} N_{1}(n, r) ;
$$


тем самым доказаны первое неравенство пункта (а) и утверждение (б).

Если набор аффинных функций $l_{i_{1}}, \ldots, l_{i_{k}} \in \mathbf{A}_{n}$ таков, что $l_{i_{u}}(x)+l_{i_{v}}(x)=1$ при всех $x \in V_{n}$ для некоторых $1 \leqslant u<v \leqslant k$, то для любой булевой функции $f \in \mathbf{F}_{2}^{V_{n}}$ справедливо равенство

$$
\rho\left(f, l_{i_{u}}\right)+\rho\left(f, l_{i_{v}}\right)=2^{n},
$$

и поэтому

$$
\max _{1 \leqslant j \leqslant k} \rho\left(f, l_{i_{j}}\right) \geqslant 2^{n-1},
$$

то есть при $r<2^{n-1}$

$$
\left|\left\{f \in \mathbf{F}_{2}^{V_{n}}: \max _{1 \leqslant j \leqslant k} \rho\left(f, l_{i_{j}}\right) \leqslant r\right\}\right|=0 .
$$

Поэтому в дальнейшем рассматриваются только наборы попарно различных аффинных булевых функций, попарные суммы которых отличны от функции, тождественно равной 1.

Далее заметим, что множество $\mathbf{A}_{n}$ аффинных функций образует в $\mathbf{F}_{2}^{V_{n}}$ группу по сложению. Поэтому для любого набора функций $l_{i_{1}}, \ldots, l_{i_{k}} \in \mathbf{A}_{n}$

$$
\left|\left\{f \in \mathbf{F}_{2}^{V_{n}}: \max _{1 \leqslant j \leqslant k} \rho\left(f, l_{i_{j}}\right) \leqslant r\right\}\right|=\left|\left\{f \in \mathbf{F}_{2}^{V_{n}}: \max _{1 \leqslant j \leqslant k} \rho\left(f \oplus l_{i_{1}}, l_{i_{j}} \oplus l_{i_{1}}\right) \leqslant r\right\}\right|,
$$

то есть при вычислении значений слагаемых в (5) достаточно рассматривать только наборы аффинных функций, содержащие нулевую функцию (поскольку $l_{i_{1}} \oplus l_{i_{1}}=0$ ).

Кроме того, если $t: V_{n} \rightarrow V_{n}-$ взаимно однозначное преобразование (например, аффинное невырожденное) и $f^{t}(x)=f(t(x))$, то $\rho(f, g)=\rho\left(f^{t}, g^{t}\right), f, g \in \mathbf{F}_{2}^{V_{n}}$, и

$$
\left|\left\{f \in \mathbf{F}_{2}^{V_{n}}: \max _{1 \leqslant j \leqslant k} \rho\left(f, l_{i_{j}}\right) \leqslant r\right\}\right|=\left|\left\{f \in \mathbf{F}_{2}^{V_{n}}: \max _{1 \leqslant j \leqslant k} \rho\left(f^{t}, l_{i_{j}}^{t}\right) \leqslant r\right\}\right| .
$$

Отсюда следует, что при вычислении значений слагаемых в (5) можно использовать замену переменных, переводящую линейно независимые аффинные функции из набора $\left\{l_{i_{2}} \oplus l_{i_{1}}, \ldots, l_{i_{k}} \oplus l_{i_{1}}\right\} \subset \mathbf{A}_{n}$ в координатные функции $l_{2}(x)=x_{1}, l_{4}(x)=x_{2}, \ldots$ Значит, все слагаемые внутренней суммы в (5), соответствующие случаю $k=2$, совпадают с $\left|\left\{f \in \mathbf{F}_{2}^{V_{n}}: \rho(f, 0) \leqslant r, \rho\left(f, l_{2}\right) \leqslant r\right\}\right|$, а слагаемые внутренней суммы в (5), соответствующие случаю $k=3$, совпадают с $\left|\left\{f \in \mathbf{F}_{2}^{V_{n}}: \rho(f, 0) \leqslant r, \rho\left(f, l_{2}\right) \leqslant r, \rho\left(f, l_{4}\right) \leqslant r\right\}\right|$.

Число ненулевых слагаемых внутренней суммы в (5) с $k=2$ равно числу пар различных аффинных функций, сумма которых отлична от функции $l_{1}(x) \equiv 1$, то есть $4\left(\begin{array}{c}2^{n} \\ 2\end{array}\right)$ (каждой паре различных линейных функций соответствует 4 пары аффинных функций).

Число ненулевых слагаемых внутренней суммы в (5) с $k=3$ равно числу троек попарно различных аффинных функций, в которых сумма любых двух функций отлична от тождественной 1 , то есть $8\left(\begin{array}{c}2^{n} \\ 3\end{array}\right)$ (каждой тройке попарно различных линейных функций соответствует 8 троек аффинных функций).

Покажем сначала, что если $l_{2}(x)=x_{1}, x=\left(x_{1}, \ldots, x_{n}\right) \in V_{n}$, и $2^{n-2} \leqslant r<2^{n-1}$, то

$$
\left|\left\{f \in \mathbf{F}_{2}^{V_{n}}: \rho(f, 0) \leqslant r, \rho\left(f, l_{2}\right) \leqslant r\right\}\right|=N_{2}(n, r) .
$$

Сопоставим каждой функции $f \in \mathbf{F}_{2}^{V_{n}}$ величины

$$
\begin{aligned}
& m_{0}=\left|\left\{x=\left(x_{1}, \ldots, x_{n}\right) \in V_{n}: x_{1}=0, f(x)=1\right\}\right|, \\
& m_{1}=\left|\left\{x=\left(x_{1}, \ldots, x_{n}\right) \in V_{n}: x_{1}=1, f(x)=1\right\}\right| .
\end{aligned}
$$


Число функций $f \in \mathbf{F}_{2}^{V_{n}}$ с фиксированными значениями $m_{0}$ и $m_{1}$ равно, очевидно, $\left(\begin{array}{c}2^{n-1} \\ m_{0}\end{array}\right)\left(\begin{array}{c}2^{n-1} \\ m_{1}\end{array}\right)$. Далее,

$$
\rho(f, 0)=m_{0}+m_{1}, \quad \rho\left(f, l_{2}\right)=m_{0}+2^{n-1}-m_{1},
$$

то есть при $2^{n-2} \leqslant r<2^{n-1}$ условия $\left\{\rho(f, 0) \leqslant r, \rho\left(f, l_{2}\right) \leqslant r\right\}$ эквивалентны условиям

$$
\left\{\begin{array} { l } 
{ 0 \leqslant m _ { 0 } , m _ { 1 } \leqslant 2 ^ { n - 1 } , } \\
{ m _ { 0 } + m _ { 1 } \leqslant r } \\
{ m _ { 0 } + 2 ^ { n - 1 } - m _ { 1 } \leqslant r }
\end{array} \Longleftrightarrow \left\{\begin{array}{l}
0 \leqslant m_{0} \leqslant r-2^{n-2}, \\
2^{n-1}-\left(r-m_{0}\right) \leqslant m_{1} \leqslant r-m_{0} .
\end{array}\right.\right.
$$

Значит,

$$
\begin{aligned}
\left|\left\{f \in \mathbf{F}_{2}^{V_{n}}: \rho(f, 0) \leqslant r, \rho\left(f, l_{2}\right) \leqslant r\right\}\right| & =N_{2}(n, r) \\
& =\sum_{m_{0}=0}^{r-2^{n-2}} \sum_{m_{1}=2^{n-1}-\left(r-m_{0}\right)}^{r-m_{0}}\left(\begin{array}{c}
2^{n-1} \\
m_{0}
\end{array}\right)\left(\begin{array}{c}
2^{n-1} \\
m_{1}
\end{array}\right) .
\end{aligned}
$$

Аналогично покажем, что при $l_{2}(x)=x_{1}, l_{4}(x)=x_{2}, x \in V_{n}$

$$
\left|\left\{f \in \mathbf{F}_{2}^{V_{n}}: \rho(f, 0) \leqslant r, \rho\left(f, l_{2}\right) \leqslant r, \rho\left(f, l_{4}\right) \leqslant r\right\}\right|=N_{3}(n, r) .
$$

Сопоставим каждой функции $f \in \mathbf{F}_{2}^{V_{n}}$ величины

$$
m_{i j}=\left|\left\{x=\left(x_{1}, \ldots, x_{n}\right) \in V_{n}: x_{1}=i, x_{2}=j, f(x)=1\right\}\right|, i, j \in\{0,1\} .
$$

Число функций $f \in \mathbf{F}_{2}^{V_{n}}$ с фиксированными значениями $m_{00}, m_{01}, m_{10}, m_{11}$ равно, очевидно, $\left(\begin{array}{c}2^{n-2} \\ m_{00}\end{array}\right)\left(\begin{array}{c}2^{n-2} \\ m_{01}\end{array}\right)\left(\begin{array}{c}2^{n-2} \\ m_{10}\end{array}\right)\left(\begin{array}{c}2^{n-2} \\ m_{11}\end{array}\right)$. Далее,

$$
\begin{aligned}
& \rho(f, 0)=m_{00}+m_{01}+m_{10}+m_{11}, \\
& \rho\left(f, l_{2}\right)=m_{00}+m_{01}+\left(2^{n-2}-m_{10}\right)+\left(2^{n-2}-m_{11}\right), \\
& \rho\left(f, l_{4}\right)=m_{00}+\left(2^{n-2}-m_{01}\right)+m_{10}+\left(2^{n-2}-m_{11}\right),
\end{aligned}
$$

то есть при $2^{n-2} \leqslant r<2^{n-1}$ условия $\left\{\rho(f, 0) \leqslant r, \rho\left(f, l_{2}\right) \leqslant r, \rho\left(f, l_{4}\right) \leqslant r\right\}$ эквивалентны условиям

$$
\begin{aligned}
0 \leqslant m_{00}, m_{01}, m_{10}, m_{11} & \leqslant 2^{n-2}, \\
m_{00}+m_{01}+m_{10}+m_{11} & \leqslant r, \\
m_{00}+m_{01}-m_{10}-m_{11}+2^{n-1} & \leqslant r, \\
m_{00}-m_{01}+m_{10}-m_{11}+2^{n-1} & \leqslant r,
\end{aligned}
$$

или, что то же самое,

$$
\begin{aligned}
0 \leqslant m_{00}, m_{01}, m_{10}, m_{11} & \leqslant 2^{n-2}, \\
m_{01}+m_{10} & \leqslant r-\left(m_{00}+m_{11}\right), \\
\left|m_{01}-m_{10}\right| & \leqslant m_{11}-m_{00}+r-2^{n-1} .
\end{aligned}
$$


Тогда при $r \geqslant 2^{n-2}$

$$
\begin{aligned}
& \left|\left\{f \in \mathbf{F}_{2}^{V_{n}}: \rho(f, 0) \leqslant r, \rho\left(f, l_{2}\right) \leqslant r, \rho\left(f, l_{4}\right) \leqslant r\right\}\right|=N_{3}(n, r) \\
& =\sum_{\substack{m_{00}, m_{11} \geqslant 0 \\
m_{00}+m_{11} \leqslant r \\
m_{11}-m_{00} \geqslant 2^{n-1}-r}}\left(\begin{array}{c}
2^{n-2} \\
m_{00}
\end{array}\right)\left(\begin{array}{c}
2^{n-2} \\
m_{11}
\end{array}\right) \sum_{\substack{m_{01}, m_{10} \geqslant 0 \\
m_{01}+m_{10} \leqslant r-\left(m_{00}+m_{11}\right) \\
m_{01}-m_{10} \leqslant r-2^{n-1}-m_{00}+m_{11}}}\left(\begin{array}{l}
2^{n-2} \\
m_{01}
\end{array}\right)\left(\begin{array}{l}
2^{n-2} \\
m_{10}
\end{array}\right) .
\end{aligned}
$$

Чтобы упростить правую часть, заметим, что область суммирования во внешней сумме можно представить в виде

$$
0 \leqslant m_{00} \leqslant r-2^{n-2}, \quad 2^{n-1}-r+2 m_{00} \leqslant m_{00}+m_{11} \leqslant r .
$$

Действительно, $0 \leqslant m_{11} \leqslant 2^{n-2}$, поэтому

$$
0 \leqslant m_{00} \leqslant m_{11}+r-2^{n-1} \leqslant r-2^{n-2} ;
$$

кроме того,

$$
2^{n-1}-r+2 m_{00} \leqslant m_{11}-m_{00}+2 m_{00}=m_{00}+m_{11} \leqslant r .
$$

Обратно, если выполняются условия (6), то

$$
\begin{aligned}
m_{00} & \leqslant r-2^{n-2} \leqslant 2^{n-2}, \\
m_{11}-m_{00} & \geqslant 2^{n-1}-r \\
m_{00}+m_{11} & \leqslant r .
\end{aligned}
$$

Используя для внутренней суммы обозначение

$$
S(a, b)=\sum_{\substack{g, h \geqslant 0 \\
g+h \leqslant a,|g-h| \leqslant b}}\left(\begin{array}{c}
2^{n-2} \\
g
\end{array}\right)\left(\begin{array}{c}
2^{n-2} \\
h
\end{array}\right),
$$

получаем указанное перед формулировкой теоремы 1 представление

$$
N_{3}(n, r)=\sum_{v=0}^{r-2^{n-2}} \sum_{u=2^{n-1}-r+2 v}^{r}\left(\begin{array}{c}
2^{n-2} \\
v
\end{array}\right)\left(\begin{array}{c}
2^{n-2} \\
u-v
\end{array}\right) S\left(r-u, r+u-2 v-2^{n-1}\right) \text {. }
$$

Нам осталось показать, что для любых попарно различных аффинных функций $l_{i_{1}}$, $l_{i_{2}}, l_{i_{3}}, l_{i_{4}}$

$$
\max _{f \in \mathbf{F}_{2}^{V n}}\left\{\rho\left(f, l_{i_{1}}\right), \rho\left(f, l_{i_{2}}\right), \rho\left(f, l_{i_{3}}\right), \rho\left(f, l_{i_{4}}\right)\right\} \geqslant 2^{n-2}+2^{n-4} .
$$

Действительно, если $l_{i_{1}}, l_{i_{2}}, l_{i_{3}}, l_{i_{4}}-$ различные аффинные функции, попарные суммы которых отличны от $l_{1}(x) \equiv 1$, то, добавляя $l_{i_{1}}$ ко всем четырем функциям и проводя подходящую замену переменных, приходим либо к набору $\left\{0, x_{1}, x_{2}, x_{3}\right\}=\left\{0, l_{2}, l_{4}, l_{8}\right\}$, либо (если $l_{i_{1}} \oplus l_{i_{2}} \oplus l_{i_{3}} \oplus l_{i_{4}}$ равно либо $l_{0} \equiv 0$, либо $\left.l_{1} \equiv 1\right)$ к набору $\left\{0, x_{1}, x_{2}, x_{1} \oplus x_{2}\right\}=$ $\left\{0, l_{2}, l_{4}, l_{6}\right\}$.

Рассмотрим сначала первый случай. Положим для $i, j, k \in\{0,1\}$

$$
m_{i j k}=\left|\left\{x=\left(x_{1}, \ldots, x_{n}\right) \in V_{n}: x_{1}=i, x_{2}=j, x_{3}=k, f(x)=1\right\}\right| \leqslant 2^{n-3} .
$$


В этих терминах условие $\max \left\{\rho(f, 0), \rho\left(f, l_{2}\right), \rho\left(f, l_{4}\right), \rho\left(f, l_{8}\right)\right\} \leqslant r$ принимает вид

$$
\begin{aligned}
& \rho(f, 0)=m_{000}+m_{001}+m_{010}+m_{011}+m_{100}+m_{101}+m_{110}+m_{111} \leqslant r, \\
& \rho\left(f, l_{2}\right)=2^{n-1}+m_{000}+m_{001}+m_{010}+m_{011}-m_{100}-m_{101}-m_{110}-m_{111} \leqslant r, \\
& \rho\left(f, l_{4}\right)=2^{n-1}+m_{000}+m_{001}-m_{010}-m_{011}+m_{100}+m_{101}-m_{110}-m_{111} \leqslant r, \\
& \rho\left(f, l_{8}\right)=2^{n-1}+m_{000}-m_{001}+m_{010}-m_{011}+m_{100}-m_{101}+m_{110}-m_{111} \leqslant r .
\end{aligned}
$$

Складывая эти неравенства почленно, получим неравенство

$$
2^{n}+2^{n-1}+4 m_{000}+2 m_{001}+2 m_{010}+2 m_{100}-2 m_{111} \leqslant 4 r ;
$$

так как $0 \leqslant m_{i j k} \leqslant 2^{n-3}$, отсюда следует, что

$$
r \geqslant 2^{n-2}+2^{n-3}-2 \cdot 2^{n-5}=2^{n-2}+2^{n-4} .
$$

Во втором случае аналогичная система отличается от рассмотренной лишь последним неравенством:

$$
\rho\left(f, l_{6}\right)=2^{n-1}+m_{000}+m_{001}-m_{010}-m_{011}-m_{100}-m_{101}+m_{110}+m_{111} \leqslant r,
$$

сложение всех 4 неравенств системы дает неравенство

$$
2^{n}+2^{n-1}+4 m_{000}+4 m_{001} \leqslant 4 r,
$$

значит,

$$
r \geqslant 2^{n-2}+2^{n-3}>2^{n-2}+2^{n-4}
$$

Последнее утверждение теоремы следует из того, что при $r<2^{n-2}+2^{n-4}$ все слагаемые в (5) с $k \geqslant 4$ равны 0.

Доказательство теоремы 3 отличается от доказательства теоремы 1 только тем, что теперь

$$
\left|\mathbf{F}_{2}^{V_{n}, 0}(r)\right|=\sum_{k=1}^{2^{n}}(-1)^{k-1} \sum_{0 \leqslant i_{1}<\ldots<i_{k}<2^{n}}\left|\left\{f \in \mathbf{F}_{2}^{V_{n}}: \max _{1 \leqslant j \leqslant k} \rho\left(f, l_{2 i_{j}}\right) \leqslant r\right\}\right|,
$$

то есть суммирование проводится по линейным (аффинным с нулевым свободным членом) функциям. Поэтому в формуле (8) по сравнению с (5) числа слагаемых во внутренних суммах при $k=1,2,3$ уменьшаются соответственно в 2, 4 и 8 раз.

Замечание 1. Входящие в формулу для $N_{3}(n, r)$ значения $S(a, b)$ удовлетворяют рекуррентным соотношениям

$$
S(0,0)=S(1,0)=1, \quad S(1,1)=1+2\left(\begin{array}{c}
2^{n-2} \\
1
\end{array}\right)=1+2^{n-1},
$$

если $a=2,3, \ldots$ и $a \equiv b(\bmod 2)$, то $0 \leqslant b \leqslant a$ и

$$
\begin{aligned}
S(a, b)=S(a, b-2) & +S(a-1, b-1)+S(a-2, b) \\
& -S(a-1, b-2)-S(a-2, b-1)+2\left(\begin{array}{c}
2^{n-2} \\
(a+b) / 2
\end{array}\right)\left(\begin{array}{c}
2^{n-2} \\
(a-b) / 2
\end{array}\right),
\end{aligned}
$$


Таблица 1.

\begin{tabular}{|c|c|r|r||c|r|r|}
\hline \multicolumn{4}{|c||}{$n=6 ;\left|\mathbf{F}_{2}^{V_{6}}\right|=2^{2^{6}} \approx 1,845 \cdot 10^{19}$} & \multicolumn{3}{c|}{$n=8 ;\left|\mathbf{F}_{2}^{V_{8}}\right|=2^{2^{8}} \approx 1,158 \cdot 10^{77}$} \\
\hline$\alpha$ & $r$ & нижняя оценка & верхняя оценка & $r$ & нижняя оценка & верхняя оценка \\
\hline $1 / 2$ & 16 & $9,129 \cdot 10^{16}$ & $9,129 \cdot 10^{16}$ & 64 & $1,451 \cdot 10^{64}$ & $1,451 \cdot 10^{64}$ \\
\hline $5 / 8$ & 20 & $4,101 \cdot 10^{18}$ & $4,105 \cdot 10^{18}$ & 80 & $5,594 \cdot 10^{70}$ & $5,594 \cdot 10^{70}$ \\
\hline $3 / 4$ & 24 & $-5,355 \cdot 10^{19}$ & $7,853 \cdot 10^{19}$ & 96 & $2,241 \cdot 10^{75}$ & $2,241 \cdot 10^{75}$ \\
\hline $13 / 16$ & 26 & $-8,598 \cdot 10^{20}$ & $2,836 \cdot 10^{21}$ & 104 & $6,165 \cdot 10^{76}$ & $6,856 \cdot 10^{76}$ \\
\hline & 22 & $1,177 \cdot 10^{19}$ & $1,336 \cdot 10^{19}$ & 105 & $6,497 \cdot 10^{76}$ & $8,981 \cdot 10^{76}$ \\
\hline & 28 & $-5,074 \cdot 10^{21}$ & $4,021 \cdot 10^{22}$ & 120 & $-4,509 \cdot 10^{80}$ & $1,389 \cdot 10^{82}$ \\
\hline
\end{tabular}

\section{Таблица 2.}

\begin{tabular}{|c|c|c|c||c|c|c|}
\hline \multicolumn{3}{|c||}{$n=10 ;\left|\mathbf{F}_{2}^{V_{10}}\right|=2^{2^{10}} \approx 1,797 \cdot 10^{308}$} & \multicolumn{3}{c|}{$n=12 ;\left|\mathbf{F}_{2}^{V_{12}}\right|=2^{2^{12}} \approx 1,044 \cdot 10^{1233}$} \\
\hline$\alpha$ & $r$ & нижняя оценка & верхняя оценка & $r$ & нижняя оценка & верхняя оценка \\
\hline $1 / 2$ & 256 & $1,062 \cdot 10^{252}$ & $1,062 \cdot 10^{252}$ & 1024 & $3,704 \cdot 10^{1002}$ & $3,704 \cdot 10^{1002}$ \\
\hline $5 / 8$ & 320 & $1,623 \cdot 10^{278}$ & $1,623 \cdot 10^{278}$ & 1280 & $1,370 \cdot 10^{1107}$ & $1,370 \cdot 10^{1107}$ \\
\hline $3 / 4$ & 384 & $2,108 \cdot 10^{296}$ & $2,108 \cdot 10^{296}$ & 1536 & $1,799 \cdot 10^{1179}$ & $1,799 \cdot 10^{1179}$ \\
\hline $7 / 8$ & 448 & $1,261 \cdot 10^{307}$ & $1,262 \cdot 10^{307}$ & 1792 & $5,559 \cdot 10^{1221}$ & $5,559 \cdot 10^{1221}$ \\
\hline & 459 & $9,505 \cdot 10^{307}$ & $1,242 \cdot 10^{308}$ & 1930 & $5,329 \cdot 10^{1232}$ & $6,875 \cdot 10^{1232}$ \\
\hline & 496 & $-1,037 \cdot 10^{313}$ & $1,175 \cdot 10^{315}$ & 2016 & $-9,236 \cdot 10^{1238}$ & $4,095 \cdot 10^{1241}$ \\
\hline
\end{tabular}

если $a=2,3, \ldots$ и $a \neq \equiv b(\bmod 2)$, то $0 \leqslant b<a$ и

$$
S(a, b)=S(a, b-1)+S(a-1, b)-S(a-1, b-1) .
$$

С помощью этих рекуррентных формул можно организовать вычисление (формально четырехкратной) суммы в $N_{3}(n, r)$ как двукратной. Тем не менее, сложность вычислений растет по $n$ экспоненциально.

Некоторые результаты вычислений по формулам (3) теоремы 2 приведены в табл. 1, 2 и 3. В этих таблицах $\alpha=r / 2^{n-1}$, предпоследние строки соответствуют максимальному значению $r$, при котором значение нижней оценки больше ее значения при $r-1$, a в последних строках значения $r=\left[2^{n-1}-2^{n / 2-1}\right]$ соответствуют расстояниям между множествами аффинных функций и бент-функций (за исключением случая $n=13$ ).

Таблицы показывают, что оценки оказываются довольно точными в неожиданно широкой области значений $r$. В следующей части статьи получены оценки, подтверждающие этот эффект.

\section{3. Упрощенные оценки числа булевых функций}

Точные формулы для величин $N_{1}(n, r), N_{2}(n, r), N_{3}(n, r)$ в теоремах 1 и 2 слишком сложны для вычислений даже при умеренных значениях $n$. Далее будут приведены простые 
Таблица 3.

\begin{tabular}{|c|c|c|c||c|c|c|}
\hline \multicolumn{3}{|c||}{$n=13 ;\left|\mathbf{F}_{2}{ }^{13}\right|=2^{2^{13}} \approx 1,090 \cdot 10^{2466}$} & \multicolumn{3}{c|}{$n=14 ;\left|\mathbf{F}_{2}^{V_{14}}\right|=2^{2^{14}} \approx 1,189 \cdot 10^{4932}$} \\
\hline$\alpha$ & $r$ & нижняя оценка & верхняя оценка & $r$ & нижняя оценка & верхняя оценка \\
\hline $1 / 2$ & 2048 & $1,097 \cdot 10^{2003}$ & $1,097 \cdot 10^{2003}$ & 4096 & $6,817 \cdot 10^{4003}$ & $6,817 \cdot 10^{4003}$ \\
\hline $3 / 4$ & 3072 & $1,743 \cdot 10^{2356}$ & $1,743 \cdot 10^{2356}$ & 6144 & $1,154 \cdot 10^{4710}$ & $1,154 \cdot 10^{4710}$ \\
\hline $15 / 16$ & 3840 & $1,454 \cdot 10^{2462}$ & $1,454 \cdot 10^{2462}$ & 7680 & $2,531 \cdot 10^{4921}$ & $2,531 \cdot 10^{4921}$ \\
\hline $61 / 64$ & 3904 & $1,875 \cdot 10^{2465}$ & $1,886 \cdot 10^{2465}$ & 7808 & $4,009 \cdot 10^{4927}$ & $4,009 \cdot 10^{4927}$ \\
\hline & 3922 & $5,523 \cdot 10^{2465}$ & $7,441 \cdot 10^{2465}$ & 7935 & $5,995 \cdot 10^{4931}$ & $7,948 \cdot 10^{4931}$ \\
\hline & 4032 & $-9,420 \cdot 10^{2471}$ & $4,126 \cdot 10^{2474}$ & 8128 & $-1,646 \cdot 10^{4939}$ & $2,886 \cdot 10^{4942}$ \\
\hline
\end{tabular}

оценки этих величин, позволяющие делать выводы о точности формул в теоремах 2, 3 при $n, r \rightarrow \infty$.

Для оценки $N_{1}(n, r)$ можно использовать следующие неравенства, являющиеся переформулировкой некоторых результатов статьи [6].

Теорема 4 ([6]). Если случайная величина $X_{N, p}$ имеет биномиальное распределение с параметрами $(N, p)$, то для всех $k \geqslant N p$

$$
\begin{aligned}
& \mathbf{P}\left\{X_{N, p} \geqslant k\right\} \geqslant \Phi(-U(k, N, p)), \\
& \mathbf{P}\left\{X_{N, p} \leqslant k\right\} \geqslant \Phi(U(k, N, p)),
\end{aligned}
$$

где Ф(·) - функиия стандартного нормального распределения, а

$$
U(k, N, p)=\sqrt{2 \ln \left(\left(\frac{k}{N p}\right)^{k}\left(\frac{N-k}{N(1-p)}\right)^{N-k}\right)} .
$$

Если $p=1 / 2$, то

$$
U\left(2^{n-1}(1+z), 2^{n}, 1 / 2\right)=\sqrt{2^{n} V(z)},
$$

где при $|z|<1$

$$
V(z)=(1-z) \ln (1-z)+(1+z) \ln (1+z)=\sum_{k=1}^{\infty} \frac{z^{2 k}}{k(2 k-1)} \geqslant z^{2} .
$$

Предложение 1. При $r<2^{n-1}$ справедливы оцуенки

$$
\begin{gathered}
2_{2^{n}} \Phi\left(-\sqrt{2^{n} V\left(1-r / 2^{n-1}\right)}\right) \leqslant N_{1}(n, r) \leqslant 2^{2^{n}} \Phi\left(-\sqrt{2^{n} V\left(1-(r+1) / 2^{n-1}\right)}\right), \\
\frac{2^{n 2^{n}}}{r^{r}\left(2^{n}-r\right)^{2^{n}-r} \sqrt{2^{n+1} \pi V\left(1-r / 2^{n-1}\right)}}\left(1-\frac{1}{2^{n} V\left(1-r / 2^{n-1}\right)}\right)<N_{1}(n, r) \\
\quad<\frac{2^{n 2^{n}}}{(r+1)^{r+1}\left(2^{n}-r-1\right)^{2^{n}-r-1} \sqrt{2^{n+1} \pi V\left(1-(r+1) / 2^{n-1}\right)}} .
\end{gathered}
$$

Доказательство. Первая двусторонняя оценка в (11) следует из тождеств

$$
\begin{aligned}
N_{1}(n, r) & =\sum_{m=0}^{r}\left(\begin{array}{l}
2^{n} \\
m
\end{array}\right)=\sum_{m=2^{n}-r}^{2^{n}}\left(\begin{array}{l}
2^{n} \\
m
\end{array}\right) \\
& =2^{2^{n}} \mathbf{P}\left\{X_{2^{n}, 1 / 2} \geqslant 2^{n}-r\right\}=2^{2^{n}}\left(1-\mathbf{P}\left\{X_{2^{n}, 1 / 2} \leqslant 2^{n}-r-1\right\}\right),
\end{aligned}
$$


теоремы 4 и связи функций $U\left(2^{n-1}(1+z), 2^{n}, 1 / 2\right)$ и $V(z)$.

Вторая двусторонняя оценка в (11) следует из первой оценки, неравенств для функции стандартного нормального распределения (см. [3], гл. 7, §1):

$$
\frac{e^{-x^{2} / 2}}{\sqrt{2 \pi}}\left(\frac{1}{x}-\frac{1}{x^{3}}\right)<\Phi(-x)=1-\Phi(x)<\frac{e^{-x^{2} / 2}}{\sqrt{2 \pi}} \frac{1}{x}, \quad x>0,
$$

и того, что

$$
\begin{aligned}
\exp \left\{-\frac{1}{2} 2^{n} V\left(1-\frac{r}{2^{n-1}}\right)\right\} & =\exp \left\{-2^{n-1}\left(\left(2-\frac{r}{2^{n-1}}\right) \ln \left(2-\frac{r}{2^{n-1}}\right)+\frac{r}{2^{n-1}} \ln \frac{r}{2^{n-1}}\right)\right\} \\
& =\left(\frac{2^{n-1}}{r}\right)^{r}\left(\frac{2^{n-1}}{2^{n}-r}\right)^{2^{n}-r}=\frac{2^{(n-1) 2^{n}}}{r^{r}\left(2^{n}-r\right)^{2^{n}-r}} .
\end{aligned}
$$

Замечание 2. Вторая оценка в (11) грубее первой, но она не содержит специальных функций. Сравним величины верхней и нижней оценок в этой формуле.

Так как $V(z) \geqslant z^{2}$, то при $r<2^{n-1}-\sqrt{2^{n-1} n \ln 2}$

$$
1>1-\frac{1}{2^{n} V\left(1-r / 2^{n-1}\right)} \geqslant 1-\frac{1}{2^{n}\left(1-r / 2^{n-1}\right)^{2}}>1-\frac{1}{2 n \ln 2},
$$

значит, отношение нижней оценки к верхней не меньше

$$
\frac{r+1}{2^{n}-r}\left(1+\frac{1}{r}\right)^{r}\left(1-\frac{1}{2^{n}-r}\right)^{2^{n}-r-1} \sqrt{\frac{V\left(1-(r+1) / 2^{n-1}\right)}{V\left(1-r / 2^{n-1}\right)}}\left(1-\frac{1}{2 n \ln 2}\right) .
$$

Функция $V(z)$ выпукла и

$$
V^{\prime}(z)=\ln \frac{1+z}{1-z}<2 \ln \frac{1}{1-z}
$$

поэтому

$$
V\left(1-\frac{r+1}{2^{n-1}}\right)>V\left(1-\frac{r}{2^{n-1}}\right)-\frac{1}{2^{n-1}} V^{\prime}\left(1-\frac{r}{2^{n-1}}\right)>V\left(1-\frac{r}{2^{n-1}}\right)-\frac{2}{2^{n-1}} \ln \frac{2^{n-1}}{r}
$$

при $1 \leqslant r<2^{n-1}-\sqrt{2^{n-1} n \ln 2}$, в силу чего

$$
1>\frac{V\left(1-(r+1) / 2^{n-1}\right)}{V\left(1-r / 2^{n-1}\right)}>1-\frac{2 \ln \left(2^{n-1} / r\right)}{2^{n-1}\left(1-r / 2^{n-1}\right)^{2}} .
$$

Функция $u(x)=(1-1 / x)^{-2} \ln x$ на $(1, \infty)$ положительна и имеет единственную точку минимума при $x \approx 3,5128$ : это единственный нуль производной

$$
u^{\prime}(x)=x(x-1)^{-3}(x-1-2 \ln x) .
$$

Поэтому максимум $u(x)$ на любом отрезке, содержащемся в $(1, \infty)$, достигается на одном из его концов. Положим

$$
R=2^{n-1}-\sqrt{2^{n-1} n \ln 2}
$$


Так как $\sqrt{2^{1-n} n \ln 2}<1 / 2$ при $n \geqslant 6$ и $-\ln (1-x) \leqslant 3 x / 2$ при $0 \leqslant x \leqslant 1 / 2$, то справедливы соотношения

$$
\begin{aligned}
\max _{1 \leqslant r \leqslant R} \frac{\ln \left(2^{n-1} / r\right)}{\left(1-r / 2^{n-1}\right)^{2}} & =\max \left\{\frac{(n-1) \ln 2}{\left(1-1 / 2^{n-1}\right)^{2}}, \frac{\ln \left(2^{n-1} / R\right)}{\left(1-R / 2^{n-1}\right)^{2}}\right\} \\
& <\max \left\{n \ln 2,-\frac{\ln \left(1-\sqrt{2^{1-n} n \ln 2}\right)}{2^{1-n} n \ln 2}\right\}<\frac{3}{2} \sqrt{2^{n-1} /(n \ln 2)},
\end{aligned}
$$

то есть при $n \geqslant 6,1 \leqslant r<2^{n-1}-\sqrt{2^{n-1} n \ln 2}$

$$
1>\frac{V\left(1-(r+1) / 2^{n-1}\right)}{V\left(1-r / 2^{n-1}\right)}>1-\frac{3}{\sqrt{n 2^{n-1} \ln 2}} .
$$

Наконец, из неравенства $\ln (1+x)>x-x^{2}$, справедливого при $|x| \leqslant 1 / 2$, следует, что при $2 \leqslant r<2^{n-1}-\sqrt{2^{n-1} n \ln 2}$ верны соотношения

$$
\begin{aligned}
\left(1+\frac{1}{r}\right)^{r}\left(1-\frac{1}{2^{n}-r}\right)^{2^{n}-r-1} & >\exp \left\{r\left(\frac{1}{r}-\frac{1}{r^{2}}\right)-\left(2^{n}-r-1\right)\left(\frac{1}{2^{n}-r}+\frac{1}{\left(2^{n}-r\right)^{2}}\right)\right\} \\
& =\exp \left\{-\frac{1}{r}+\frac{1}{2^{n}-r}\right\} .
\end{aligned}
$$

Таким образом, если $r, n \rightarrow \infty$, то величина (13), которая является нижней оценкой отношения левой и правой частей $(11)$, стремится к $(r+1) /\left(2^{n}-r\right)$; эта дробь равна отношению $\left(\begin{array}{c}2^{n} \\ r\end{array}\right) /\left(\begin{array}{c}2^{n} \\ r+1\end{array}\right)$ максимальных слагаемых в суммах, представляющих $N_{1}(n, r)$ и $N_{1}(n, r+1)$ соответственно. Значит, неравенства (11) дают правильное представление о величине $N_{1}(n, r)$.

Нам потребуются также более простые и грубые оценки для $N_{1}(n, r)$ и $N_{2}(n, r)$.

Предложение 2. Если $0 \leqslant r \leqslant 2^{n-1}-\sqrt{2^{n-1} n \ln 2}$, то

$$
\begin{gathered}
\left(\begin{array}{c}
2^{n} \\
r
\end{array}\right)(1+q) \leqslant N_{1}(n, r) \leqslant\left(\begin{array}{c}
2^{n} \\
r
\end{array}\right) /(1-q), \\
\left(\begin{array}{c}
2^{n-1} \\
2^{n-2}
\end{array}\right) N_{1}\left(n-1, r-2^{n-2}\right) \leqslant N_{2}(n, r) \leqslant\left(\begin{array}{c}
2^{n-1} \\
2^{n-2}
\end{array}\right)\left(\begin{array}{c}
2^{n-1} \\
r-2^{n-2}
\end{array}\right) \frac{1+q_{*}}{\left(1-q_{*}\right)^{2}},
\end{gathered}
$$

2de

$$
q=\frac{r}{2^{n}-r+1}, \quad q_{*}=\frac{r-2^{n-2}}{2^{n-1}+2^{n-2}-r+1}<q .
$$

Доказательство. Для любого натурального $M$ отношение

$$
q(M, k)=\frac{\left(\begin{array}{c}
M \\
k-1
\end{array}\right)}{\left(\begin{array}{c}
M \\
k
\end{array}\right)}=\frac{k}{M-k+1}
$$

возрастает с ростом $k$, поэтому при $q=q\left(2^{n}, r\right)$

$$
\begin{aligned}
& N_{1}(n, r)=\sum_{m=0}^{r}\left(\begin{array}{c}
2^{n} \\
m
\end{array}\right) \geqslant\left(\begin{array}{c}
2^{n} \\
r
\end{array}\right)+\left(\begin{array}{c}
2^{n} \\
r-1
\end{array}\right)=\left(\begin{array}{c}
2^{n} \\
r
\end{array}\right)(1+q), \\
& N_{1}(n, r) \leqslant\left(\begin{array}{c}
2^{n} \\
r
\end{array}\right) \sum_{m=0}^{r} q^{m}<\left(\begin{array}{c}
2^{n} \\
r
\end{array}\right) /(1-q) .
\end{aligned}
$$


Чтобы получить верхнюю оценку для $N_{2}(n, r)$, заменим все слагаемые во внутренней сумме их максимальным значением $\left(\begin{array}{c}2^{n-1} \\ 2^{n-2}\end{array}\right)$, положим $q_{*}=q\left(2^{n-1}, r-2^{n-2}\right)$ и используем оценки $\left(\begin{array}{c}2^{n-1} \\ r-2^{n-2}-m\end{array}\right)<q_{*}^{m}\left(\begin{array}{c}2^{n-1} \\ r-2^{n-2}\end{array}\right)$ :

$$
\begin{aligned}
N_{2}(n, r) & =\sum_{m_{0}=0}^{r-2^{n-2}}\left(\begin{array}{c}
2^{n-1} \\
m_{0}
\end{array}\right) \sum_{m_{1}=2^{n-1}-\left(r-m_{0}\right)}^{r-m_{0}}\left(\begin{array}{c}
2^{n-1} \\
m_{1}
\end{array}\right) \\
& <\left(\begin{array}{c}
2^{n-1} \\
2^{n-2}
\end{array}\right) \sum_{m=0}^{r-2^{n-2}}\left(\begin{array}{c}
2^{n-1} \\
r-2^{n-2}-m
\end{array}\right)(1+2 m) \\
& <\left(\begin{array}{l}
2^{n-1} \\
2^{n-2}
\end{array}\right)\left(\begin{array}{c}
2^{n-1} \\
r-2^{n-2}
\end{array}\right) \sum_{m=0}^{\infty} q_{*}^{m}(1+2 m) \\
& =\left(\begin{array}{l}
2^{n-1} \\
2^{n-2}
\end{array}\right)\left(\begin{array}{c}
2^{n-1} \\
r-2^{n-2}
\end{array}\right) \frac{1+q_{*}}{\left(1-q_{*}\right)^{2}} .
\end{aligned}
$$

Оценку снизу для $N_{2}(n, r)$ можно получить, заменяя внутреннюю сумму одним максимальным слагаемым:

$$
N_{2}(n, r)>\left(\begin{array}{c}
2^{n-1} \\
2^{n-2}
\end{array}\right) \sum_{m_{0}=0}^{r-2^{n-2}}\left(\begin{array}{c}
2^{n-1} \\
m_{0}
\end{array}\right)=\left(\begin{array}{l}
2^{n-1} \\
2^{n-2}
\end{array}\right) N_{1}\left(n-1, r-2^{n-2}\right) .
$$

Наконец, неравенство $q_{*}<q$ следует из того, что

$$
\begin{aligned}
q_{*} & =\frac{r-2^{n-2}}{2^{n-1}+2^{n-2}-r+1} \\
& =1-\frac{2^{n}-2 r+1}{2^{n-1}+2^{n-2}-r+1} \\
& <1-\frac{2^{n}-2 r+1}{2^{n}-r+1} \\
& =\frac{r}{2^{n}-r+1}=q .
\end{aligned}
$$

Предложение 2 доказано.

Доказательство теоремь 1. Согласно теоремам 2 и 3

$$
\begin{gathered}
2^{n+1} N_{1}(n, r)-4\left(\begin{array}{c}
2^{n} \\
2
\end{array}\right) N_{2}(n, r) \leqslant\left|\mathbf{F}_{2}^{V_{n}}(r)\right| \leqslant 2^{n+1} N_{1}(n, r) \\
2^{n} N_{1}(n, r)-\left(\begin{array}{c}
2^{n} \\
2
\end{array}\right) N_{2}(n, r) \leqslant\left|\mathbf{F}_{2}^{V_{n}, 0}(r)\right| \leqslant 2^{n} N_{1}(n, r),
\end{gathered}
$$

поэтому при

$$
Q(n, r)=\frac{4\left(\begin{array}{c}
2^{n} \\
2
\end{array}\right) N_{2}(n, r)}{2^{n+1} N_{1}(n, r)}<\frac{2^{n} N_{2}(n, r)}{N_{1}(n, r)},
$$


получаем утверждения теоремы 1 , а именно,

$$
\begin{gathered}
2^{n+1} N_{1}(n, r)(1-Q(n, r)) \leqslant\left|\mathbf{F}_{2}^{V_{n}}(r)\right| \leqslant 2^{n+1} N_{1}(n, r), \\
2^{n} N_{1}(n, r)\left(1-\frac{1}{2} Q(n, r)\right) \leqslant\left|\mathbf{F}_{2}^{V_{n}, 0}(r)\right| \leqslant 2^{n} N_{1}(n, r) .
\end{gathered}
$$

Для завершения доказательства остается обосновать оценки величины $Q(n, r)$.

Из теорем 2 и 3 следует, что $Q(n, r)=0$ при $0 \leqslant r<2^{n-2}$.

Теорема 5. Если

$$
n \geqslant 8, \quad r=2^{n-1}-c_{r} \sqrt{n 2^{n-1} \ln 2} \geqslant 0, \quad c_{r}>1,
$$

mo

$$
Q(n, r)<\frac{1}{15} 2^{-\left(c_{r}^{2}-3 / 2\right) n} \exp \left\{\frac{2\left(c_{r}^{2} n\right)^{3 / 2}}{2^{n / 2}}\right\}
$$

Доказательство. Из предложения 2 и того, что

$$
\begin{aligned}
1-q_{*} & =\frac{2^{n}-2 r+1}{3 \cdot 2^{n-2}-r+1} \\
& >\frac{2^{n}-2 r}{3 \cdot 2^{n-2}-r}, \quad r \geqslant 2^{n-2},
\end{aligned}
$$

следует оценка

$$
\begin{aligned}
Q(n, r) & <\frac{2^{n}\left(\begin{array}{c}
2^{n-1} \\
2^{n-2}
\end{array}\right)\left(\begin{array}{c}
2^{n-1} \\
r-2^{n-2}
\end{array}\right)\left(1+q_{n, r}\right) /\left(1-q_{n, r}\right)^{2}}{\left(\begin{array}{c}
2^{n} \\
r
\end{array}\right)(1+q)} \\
& <\frac{2^{n}\left(\begin{array}{c}
2^{n-1} \\
2^{n-2}
\end{array}\right)\left(\begin{array}{c}
2^{n-1} \\
r-2^{n-2}
\end{array}\right)}{\left(\begin{array}{c}
2^{n} \\
r
\end{array}\right)}\left(\frac{3 \cdot 2^{n-2}-r}{2^{n}-2 r}\right)^{2} .
\end{aligned}
$$

Из тождеств

$$
\begin{aligned}
\left(\begin{array}{c}
2^{n} \\
r
\end{array}\right) & =\left(\begin{array}{c}
2^{n} \\
2^{n-1}
\end{array}\right) \prod_{k=1}^{2^{n-1}-r} \frac{r+k}{2^{n-1}+k}, \\
\left(\begin{array}{c}
2^{n-1} \\
r-2^{n-2}
\end{array}\right) & =\left(\begin{array}{c}
2^{n-1} \\
2^{n-2}
\end{array}\right) \prod_{k=1}^{2^{n-1}-r} \frac{r-2^{n-2}+k}{2^{n-2}+k}
\end{aligned}
$$

получаем равенство

$$
\frac{\left(\begin{array}{c}
2^{n-1} \\
r-2^{n-2}
\end{array}\right)\left(\begin{array}{c}
2^{n-1} \\
2^{n-2}
\end{array}\right)}{\left(\begin{array}{c}
2^{n} \\
r
\end{array}\right)}=\frac{\left(\left(\begin{array}{c}
2^{n-1} \\
2^{n-2}
\end{array}\right)\right)^{2}}{\left(\begin{array}{c}
2^{n} \\
2^{n-1}
\end{array}\right)} \prod_{k=1}^{2^{n-1}-r} \frac{\left(2^{n-1}+k\right)\left(r-2^{n-2}+k\right)}{(r+k)\left(2^{n-2}+k\right)}
$$

Оценим с помощью уточненной формулы Стирлинга (см. [3], гл. 2, §9)

$$
\sqrt{2 \pi m}\left(\frac{m}{e}\right)^{m} e^{1 /(12 m+1)}<m !<\sqrt{2 \pi m}\left(\frac{m}{e}\right)^{m} e^{1 /(12 m)}
$$


первую дробь в правой части (15):

$$
\begin{aligned}
\frac{\left(\begin{array}{c}
2^{n-1} 2^{n-2} \\
2^{2}
\end{array}\right.}{\left(\begin{array}{c}
2^{n} \\
2^{n-1}
\end{array}\right)}= & \frac{\left(2^{n-1} !\right)^{4}}{2^{n !}\left(2^{n-2} !\right)^{4}} \\
< & \frac{\left(2^{(n-1) / 2} 2^{(n-1) 2^{n-1}}\right)^{4}}{\sqrt{2 \pi} 2^{n / 2} 2^{n 2^{n}}\left(2^{n / 2-1} 2^{(n-2) 2^{n-2}}\right)^{4}} \\
& \times \exp \left\{\frac{4}{12 \cdot 2^{n-1}}-\frac{1}{12 \cdot 2^{n}+1}-\frac{4}{12 \cdot 2^{n-2}+1}\right\} \\
< & \frac{1}{\sqrt{2^{n-3} \pi}} e^{1 /\left(3 \cdot 2^{n-1}\right)}<\frac{8}{5 \cdot 2^{n / 2}}, \quad n \geqslant 8 .
\end{aligned}
$$

Полагая $y=2^{n-1}-r$, находим, что

$$
\begin{aligned}
\frac{\left(2^{n-1}+k\right)\left(r-2^{n-2}+k\right)}{(r+k)\left(2^{n-2}+k\right)} & =\frac{\left(2^{n-1}+k\right)\left(2^{n-2}+k\right)-y\left(2^{n-1}+k\right)}{\left(2^{n-1}+k\right)\left(2^{n-2}+k\right)-y\left(2^{n-2}+k\right)} \\
& =\frac{1-y /\left(2^{n-2}+k\right)}{1-y /\left(2^{n-1}+k\right)} \\
& =1+\left(\frac{y}{2^{n-1}+k}-\frac{y}{2^{n-2}+k}\right) \frac{1}{1-y /\left(2^{n-1}+k\right)} \\
& =1-\frac{y 2^{n-2}}{\left(2^{n-2}+k\right)\left(2^{n-1}+k-y\right)} .
\end{aligned}
$$

Таким образом, сомножители произведения в (15) строго возрастают по $k$. Поэтому

$$
\prod_{k=1}^{2^{n-1}-r} \frac{\left(2^{n-1}+k\right)\left(r-2^{n-2}+k\right)}{(r+k)\left(2^{n-2}+k\right)} \leqslant\left(\frac{2^{n}-r}{2^{n-1}+2\left(2^{n-1}-r\right)}\right)^{2^{n-1}-r}
$$

Объединение полученных оценок приводит к неравенству

$$
\begin{aligned}
Q(n, r) & <\frac{8 \cdot 2^{n / 2}}{5}\left(\frac{2^{n}-r}{2^{n-1}+2\left(2^{n-1}-r\right)}\right)^{2^{n-1}-r}\left(\frac{3 \cdot 2^{n-2}-r}{2^{n}-2 r}\right)^{2} \\
& =\frac{2 \cdot 2^{n / 2}}{5}\left(1-\frac{y}{2^{n-1}+2 y}\right)^{y}\left(\frac{2^{n-2}}{y}+1\right)^{2}
\end{aligned}
$$

где, по условию,

$$
y=2^{n-1}-r=c_{r} \sqrt{n 2^{n-1} \ln 2}, \quad 1<c_{r} \leqslant \frac{1}{2} \sqrt{2^{n-1} /(n \ln 2)} .
$$

Тогда при $n \geqslant 8$

$$
\left(\frac{2^{n-2}}{y}+1\right)^{2}<\left(\frac{2^{n / 2}}{\sqrt{2 n \ln 2}}+1\right)^{2}<\frac{1}{6} 2^{n}
$$


и

$$
\begin{aligned}
\left(1-\frac{y}{2^{n-1}+2 y}\right)^{y} & <\exp \left\{-\frac{y^{2}}{2^{n-1}+2 y}\right\} \\
& <\exp \left\{-\frac{c_{r}^{2} n \ln 2}{1+c_{r} \sqrt{5 n 2^{-n}}}\right\} \\
& <2^{-c_{r}^{2} n} \exp \left\{\frac{2\left(c_{r}^{2} n\right)^{3 / 2}}{2^{n / 2}}\right\} .
\end{aligned}
$$

Следовательно, при $n \geqslant 8$ и $r=2^{n-1}-c_{r} \sqrt{n 2^{n-1} \ln 2}, c_{r}>1$,

$$
Q(n, r)<\frac{1}{15} 2^{-\left(c_{r}^{2}-3 / 2\right) n} \exp \left\{\frac{2\left(c_{r}^{2} n\right)^{3 / 2}}{2^{n / 2}}\right\}
$$

Теорема 5 доказана.

Доказательство следствия 1. Согласно определению $x(n, r)$

$$
x(n, r)=\frac{1}{2^{n-1}}\left(2^{n-1}-r\right)^{2}-n \ln 2+\frac{1}{2} \ln (4 \pi n \ln 2) .
$$

Поэтому при $n \rightarrow \infty$

$$
e^{-x(n, r)}=\frac{2^{n}}{\sqrt{4 \pi n \ln 2}} e^{-\left(2^{n-1}-r\right)^{2} / 2^{n-1}} \rightarrow 0,
$$

если $\left(2^{n-1}-r\right)^{2} / 2^{n-1}-n \ln 2 \rightarrow \infty$, а последнее условие выполняется, если при $n \rightarrow \infty$ существует такое $\varepsilon>0$, что

$$
0 \leqslant r<2^{n-1}-(1+\varepsilon) \sqrt{2^{n-1} n \ln 2} .
$$

Значит, при условиях (16) и достаточно больших $n$

$$
\begin{aligned}
1-e^{-e^{-x(n, r)}} & >e^{-x(n, r)} \sqrt{4 / 4,5} \\
& =\frac{2^{n}}{\sqrt{4,5 \pi n \ln 2}} e^{-\left(2^{n-1}-r\right)^{2} / 2^{n-1}} .
\end{aligned}
$$

С другой стороны, из (11), (12) и неравенства $V(z) \geqslant z^{2}$ получаем оценку

$$
\begin{aligned}
N_{1}(n, r) & <2^{2^{n}} \frac{e^{-2^{n-1} V\left(1-(r+1) / 2^{n-1}\right)}}{\sqrt{2 \pi}} \frac{1}{\sqrt{2^{n} V\left(1-(r+1) / 2^{n-1}\right)}} \\
& \leqslant \frac{2^{2^{n}-n / 2}}{\sqrt{2 \pi}} \frac{2^{n-1}}{\left(2^{n-1}-r-1\right)} e^{-2^{n-1}\left(2^{n-1}-r-1\right)^{2} / 2^{2 n-2}} \\
& =\frac{2^{2^{n}+n / 2-3 / 2}}{\sqrt{\pi}\left(2^{n-1}-r-1\right)} e^{-\left(2^{n-1}-r-1\right)^{2} / 2^{n-1}} \cdot
\end{aligned}
$$


Так как $\left|\mathbf{F}_{2}^{V_{n}, 0}(r)\right|<2^{n} N_{1}(n, r)$, из (17) и последнего неравенства следует, что при условиях (16) и достаточно больших $n$

$$
\begin{aligned}
\frac{\left|\mathbf{F}_{2}^{V_{n}, 0}(r)\right|}{2^{2^{n}}\left(1-e^{-e^{-x(n, r)}}\right)} & <\frac{2^{2^{n}+3 n / 2-1} e^{-\left(2^{n-1}-r-1\right)^{2} / 2^{n-1}} / \sqrt{2 \pi}\left(2^{n-1}-r-1\right)}{2^{2^{n}+n} e^{-\left(2^{n-1}-r\right)^{2} / 2^{n-1}} / \sqrt{4,5 \pi n \ln 2}} \\
& <\frac{(3 / 2) \sqrt{2^{n} n \ln 2}}{2\left(2^{n-1}-r-1\right)} e^{\left(2^{n}-2 r\right) / 2^{n-1}} \\
& <5 / 4 \frac{\exp \left\{2\left(1-r / 2^{n-1}\right)\right\}}{2^{n / 2}\left(1-r / 2^{n-1}\right)} \sqrt{n}
\end{aligned}
$$

Следствие доказано.

\section{Список литературы}

1. Логачев О. А., Сальников А. А., Ященко В. В., Булевы функиии в теории кодирования и криптологии. МЦНМО, Москва, 2004.

2. Ryazanov B. V., Probabilistic methods in the theory of approximation of discrete functions. In: Probabilistic Methods of Discrete Mathematics. TVP/VSP, Moscow, 1993, pp. 403-412.

3. Феллер В., Введение в теорию вероятностей и ее приложения, 1. Мир, Москва, 1984.

4. Чечета С. И., О предельном распределении расстояния между случайным вектором и некоторыми двоичными кодами. Проблемы передачи информачии (1995) 31, №1, 90-98.

5. Рязанов Б. В., Чечета С. И., О приближении случайной булевой функции множеством квадратичных форм. Дискретная математика (1995) 7, №3, 129-145.

6. Alfers D., Dinges H., A normal approximation for Beta and Gamma tail probabilities. Z. Wahrscheinlichkeitstheorie verw. Gebiete (1984) 65, 399-420.

Статья поступила 19.04.2010. Переработанный вариант поступил 4.05.2010. 